\title{
Front Matter: Volume 11192
}

, "Front Matter: Volume 11192," Proc. SPIE 11192, Real-time Photonic Measurements, Data Management, and Processing IV, 1119201 (19

December 2019); doi: 10.1117/12.2560569

SPIE. Event: SPIE/COS Photonics Asia, 2019, Hangzhou, China 


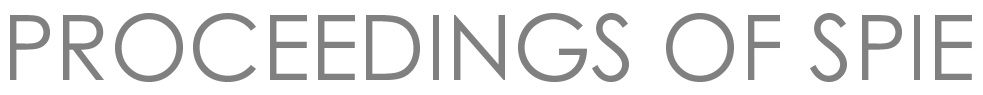

\title{
Real-time Photonic Measurements, Data Management, and Processing IV
}

\author{
Ming Li \\ Bahram Jalali \\ Mohammad Hossein Asghari \\ Editors
}

\section{2-23 October 2019 Hangzhou, China}

Sponsored by

SPIE

cos-Chinese Optical Society

\section{Cooperating Organizations}

Tsinghua University (China) • Peking University (China) • University of Science and Technology of China (China) • Zhejiang University (China) - Tianjin University (China) - Beijing Institute of Technology (China) • Beijing University of Posts and Telecommunications (China) - Nankai University (China) • Changchun University of Science and Technology (China) • University of Shanghai for Science and Technology (China) - Capital Normal University (China) • Huazhong University of Science and Technology (China) - Beijing Jiaotong University (China) • China Jiliang University (China) • Shanghai Institute of Optics and Fine Mechanics, CAS (China) - Changchun Institute of Optics, Fine Mechanics and Physics, CAS (China) • Institute of Semiconductors, CAS (China) • Institute of Optics and Electronics, CAS (China) • Institute of Physics, CAS (China) - Shanghai Institute of Technical Physics, CAS (China) - China Instrument and Control Society (China) • Japan Optical Society (Japan) • Korea Optical Society (Korea, Republic of) • Australia Optical Society (Australia) • Singapore Optical Society (Singapore) • European Optical Society

Supporting Organizations

China Association for Science and Technology (CAST) (China)

Department of Information of National Nature Science Foundation, China (NSFC) (China)

Published by

SPIE

Volume 11192 
The papers in this volume were part of the technical conference cited on the cover and title page. Papers were selected and subject to review by the editors and conference program committee. Some conference presentations may not be available for publication. Additional papers and presentation recordings may be available online in the SPIE Digital Library at SPIEDigitallibrary.org.

The papers reflect the work and thoughts of the authors and are published herein as submitted. The publisher is not responsible for the validity of the information or for any outcomes resulting from reliance thereon.

Please use the following format to cite material from these proceedings:

Author(s), "Title of Paper," in Real-time Photonic Measurements, Data Management, and Processing IV, edited by Ming Li, Bahram Jalali, Mohammad Hossein Asghari, Proceedings of SPIE Vol. 11192 (SPIE, Bellingham, WA, 2019) Seven-digit Article CID Number.

ISSN: 0277-786X

ISSN: 1996-756X (electronic)

ISBN: 9781510631014

ISBN: 9781510631021 (electronic)

Published by

SPIE

P.O. Box 10, Bellingham, Washington 98227-0010 USA

Telephone +1 3606763290 (Pacific Time) · Fax +1 3606471445

SPIE.org

Copyright @ 2019, Society of Photo-Optical Instrumentation Engineers.

Copying of material in this book for internal or personal use, or for the internal or personal use of specific clients, beyond the fair use provisions granted by the U.S. Copyright Law is authorized by SPIE subject to payment of copying fees. The Transactional Reporting Service base fee for this volume is $\$ 21.00$ per article (or portion thereof), which should be paid directly to the Copyright Clearance Center (CCC), 222 Rosewood Drive, Danvers, MA 01923. Payment may also be made electronically through CCC Online at copyright.com. Other copying for republication, resale, advertising or promotion, or any form of systematic or multiple reproduction of any material in this book is prohibited except with permission in writing from the publisher. The CCC fee code is $0277-$ $786 \times / 19 / \$ 21.00$.

Printed in the United States of America by Curran Associates, Inc., under license from SPIE.

Publication of record for individual papers is online in the SPIE Digital Library.

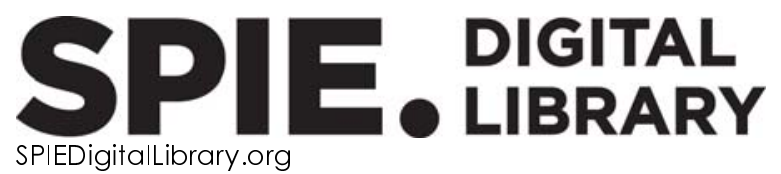

Paper Numbering: Proceedings of SPIE follow an e-First publication model. A unique citation identifier (CID) number is assigned to each article at the time of publication. Utilization of CIDs allows articles to be fully citable as soon as they are published online, and connects the same identifier to all online and print versions of the publication. SPIE uses a seven-digit CID article numbering system structured as follows:

- The first five digits correspond to the SPIE volume number.

- The last two digits indicate publication order within the volume using a Base 36 numbering system employing both numerals and letters. These two-number sets start with 00, 01, 02, 03, 04, 05, 06, 07, 08, 09, 0A, OB ... 0Z, followed by 10-1Z, 20-2Z, etc. The CID Number appears on each page of the manuscript. 


\title{
Contents
}

\author{
iii Authors \\ iv Conference Committee \\ vii Symposium Page
}

SESSION 1 OPTOELECTRONIC DEVICES AND APPLICATIONS

1119203 The dual-wavelength DFB laser based on reconstruction-equivalent-chirp technology [11192-2]

$1119204 \quad$ High-speed secure optical communication with physical random temporal encryption [11192-3]

1119207 A rapidly wavelength-switching DFB diode laser array integrated with a SOA [1 $1192-6]$

$1119208 \quad$ Electrically driven plasmonic antenna (Invited Paper) [1 11 192-8]

\section{SESSION 2 MICROWAVE PHOTONICS FOR MEASUREMENT}

11192 OC Multifunctional microwave signal generation and processing based on equivalent phase modulation (Invited Paper) [1 $1192-11$ ]

$111920 \mathrm{~A} \quad$ A triple-frequency microwave photonic link based on a polarization-multiplexing dual-parallel Mach-Zehnder modulator [1 $11192-15]$

$11192 \mathrm{OH} \quad$ Performance analysis of photonic RF self-interference cancellation for full-duplex communication [1 $11192-17$ ]

SESSION $3 \quad$ FIBER OPTIC SENSING AND MEASUREMENT

11192 OK Polarization characterization based on multidimensional Stokes vectors applied in aerosol identification [1 11 192-20]

$11192 \mathrm{OL} \quad$ Study on correlation matrix composed of multidimensional polarization index applied in aerosol recognition in the air [11192-21]

11192 ON Deep learning with synthetic photonic lattices for equalization in optical transmission systems [1 $11192-24]$ 
$1119200 \quad$ Accurate locating method of vibration sensing system based on $\varphi$-OTDR and 3D printing sensor (Invited Paper) [1 $11192-25]$

\section{SESSION 4 ULTRAFAST OPTICAL SPECTROSCOPY AND MEASUREMENT}

$111920 Q \quad$ A comparison of image recognition algorithms for cell phenotyping in optofluidic time-stretch microscopy (Invited Paper) [11 192-26]

11192 OR S Single-pixel imaging by using display illumination [1 $1192-27]$

11192 OS Ultrafast time-encoded flow imaging for Giardia cysts and Cryptosporidium oocysts detection [1 $1192-28]$

11192 OT FPGA-based real-time signal triggered storage system for ultrafast imaging flow cytometry [11192-29]

\section{POSTER SESSION}

11192 OX Multilongitudinal mode fiber laser sensor using software radio demodulation [1 $1192-22]$

11192 OY A robust target recognition and tracking panoramic surveillance system based on deep learning [1 $11192-33]$

$1119202 \quad$ An in-situ monitoring system for nitrate based on ultraviolet absorption spectrum and partial least squares [1 $11192-34]$

$1119210 \quad$ Low cost test system for silicon photonics testing [11192-35]

1119211 A fast moving object recognition method for video surveillance system [11192-36]

$1119212 \quad$ A microwave mixer with mixing spurs suppression based on a funable microwave photonic filter [1 $11192-37]$

1119213 Photonic-assisted microwave spectrum sensing based on optical carrier-suppressed singlesideband modulation and coherent detection [11192-38]

1119214 A tunable dual-frequency optoelectronic oscillator based on stimulated Brillouin scattering [1 $11192-39]$ 


\title{
Authors
}

Numbers in the index correspond to the last two digits of the seven-digit citation identifier (CID) article numbering system used in Proceedings of SPIE. The first five digits reflect the volume number. Base 36 numbering is employed for the last two digits and indicates the order of articles within the volume. Numbers start with 00, 01, 02, 03, 04, 05, 06, 07, 08, 09, OA, 0B...0Z, followed by 10-1Z, 20-2Z, etc.

\author{
Cai, Zong-Qi, $0 Z$ \\ Chen, Fei, OL \\ Chen, Hongwei, OQ, OS, OT \\ Chen, Minghua, OS, OT \\ Chen, $\mathrm{Na}, 0 \mathrm{O}$ \\ Chen, Xiangfei, 03, 07, 0X \\ Chen, Yang, $0 \mathrm{C}, 13$ \\ Chen, Zhen, 00 \\ Churkin, Dmitriy $\vee .$, ON \\ Dai, Pan, OX \\ Fan, Qiang, OY \\ Fan, Qiang, 11 \\ Fang, Tao, 07 \\ Feng, Wei-Wei, $\mathrm{OZ}$ \\ $\mathrm{Fu}$, Shuanglin, $\mathrm{OH}$ \\ Goda, Keisuke, OQ \\ Gu, Yiying, $\mathrm{OH}$ \\ Guo, Qingfeng, 12 \\ Guo, Yingxue, OS \\ Han, Xiuyou, $\mathrm{OH}$ \\ Hou, Yao-Bin, $\mathrm{OZ}$ \\ Huang, Honghao, OQ, OT \\ Jiang, Ning, 04 \\ Lei, Bo, 11 \\ Lei, Cheng, $0 Q$ \\ Li, Bin, 10 \\ Li, Fan, 14 \\ Li, Kexin, OG \\ Li, Lianyan, 03 \\ Li, Peili, 14 \\ Li, TingTing, 10 \\ Li, Zhihua, 10 \\ Liao, Riwei, OK \\ Lin, Fujiang, 10 \\ Lin, Kanglong, 07 \\ Liu, Huanhuan, 00 \\ Liu, Jialin, 13 \\ Liu, Kui, 07 \\ Liu, Ruonan, 10 \\ Liu, Shangjing, OX \\ Liv, Shiqin, 04 \\ Liu, Yingjian, 08 \\ Liu, Zhichao, 00 \\ Long, Xuan, 03 \\ LU, YUn, OS \\ Meng, Weizhe, OT \\ Pang, Fufei, 00 \\ Pankov, Artem $\mathrm{V}$.. ON \\ Qin, Cui, 12, 14
}

Qin, Jin, 08

Qiu, Kun, 04

Shen, Jianping, 03

Sidelnikov, Oleg S., ON

$\mathrm{Su}$, Xinxin, $\mathrm{OH}$

Sukhorukov, Andrey A., ON

Tan, Hai, 11

Tang, Bo, 10

Tian, Yun-fei, OR

Vatnik, llya D., ON

Wang, Chao, $\mathrm{OH}$

Wang, Dayong, $0 G$

Wang, Liang, 08

Wang, Pengyi, $\mathrm{OH}$

Wang, Shuo, $\mathrm{OH}$

Wang, Yunxin, OG

Weng, Beiyue, OC

$\mathrm{Wu}$, Zhenlin, $\mathrm{OH}$

$\mathrm{XU}$, Enming, 12, 14

$X \cup$, Ning, 03

Xu, Qihang, 14

Yang, Dengcai, OG

Yang, Feng, OG

Yang, Sigang, OS, OT

Yang, $\mathrm{Xu}, \mathrm{OH}$

Yang, Yan, 10

Yuan, Bocheng, 03

Zeng, Nan, OK, OL

Zhang, Hongbiao, OG

Zhang, Liang, 00

Zhang, Peng, 10

Zhang, Yiqun, 04

Zhang, Yunshan, 03

Zhang, Zuxing, 12, 14

Zhao, Anke, 04

Zhao, Mingshan, $\mathrm{OH}$

Zhao, Wanyue, OQ, OS, OT

Zhou, Xiaohong, OS

Zhou, Yu, OX

Zou, ErBo, OY

Zou, Hui, 03 
Proc. of SPIE Vol. 11192 1119201-6

Downloaded From: https://www.spiedigitallibrary.org/conference-proceedings-of-spie on 26 Apr 2023 Terms of Use: https://www.spiedigitallibrary.org/terms-of-use 


\title{
Symposium Committees
}

\author{
General Chairs
}

Jim M. Oschmann, President, SPIE and Ball Aerospace (United States)

Qihuang Gong, President, Chinese Optical Society and Peking University (China)

General Co-chairs

Guangcan Guo, Past President, Chinese Optical Society and University of Science and Technology of China (China)

Zejin Liu, Vice President, Chinese Optical Society and National University of Defense Technology (China)

Technical Program Chairs

Ruxin Li, Vice President, Chinese Optical Society and Shanghai Institute of Optics and Fine Mechanics (China)

Xingde Li, Johns Hopkins University (United States)

Technical Program Co-chairs

Tianchu Li, National Institute of Metrology (China)

Wei Huang, Northwestern Polytechnical University (China)

Ying Gu, Vice President, Chinese Optical Society and PLA General Hospital (China)

Huilin Jiang, Changchun University of Science and Technology (China)

Wenqing Liu, Vice President, Chinese Optical Society, and Anhui Institute of Optics and Fine Mechanics (China)

Guobin Fan, China Academy of Engineering Physics (China)

Suotang Jia, Vice President, Chinese Optical Society, and Shanxi University (China)

Xiaomin Ren, Vice President, Chinese Optical Society, and Beijing University of Posts and Telecommunications (China)

Secretaries-General

Bo Gu, Deputy Secretary General, Chinese Optical Society (China)

Hong Yang, Deputy Secretary General, Chinese Optical Society and Peking University (China) 
Yan Li, Deputy Secretary General, Chinese Optical Society, and Peking University (China)

Daoxin Dai, Zhejiang University (China)

Local Organizing Committee Chair

Xu Liu, Secretary General, Chinese Optical Society and Zhejiang University (China)

Local Organizing Committee Co-chairs

Jianrong Qiu, Zhejiang University (China)

Daoxin Dai, Zhejiang University (China)

Local Secretaries

Wei Xiong, Chinese Optical Society (China)

Qing Yang, Zhejiang University (China)

Local Organizing Committee

Qing Yang, Zhejiang University (China)

Lan Wu, Zhejiang University (China)

Yaocheng Shi, Zhejiang University (China)

Dong Liu, Zhejiang University (China)

Yungui Ma, Zhejiang University (China)

Ke Si, Zhejiang University (China)

Yang Yang, Zhejiang University (China)

Xinyong Dong, China Jiliang University (China)

Le Wang, China Jiliang University (China)

Fei Tong, Chinese Optical Society (China)

Technical Organizing Committee

Mohammad Hossein Asghari, Loyola Marymount University

(United States) and Tachyonics Inc. (United States)

Pablo Benítez, Universidad Politécnica de Madrid (Spain)

Liangcai Cao, Tsinghua University (China)

P. Scott Carney, University of Rochester (United States)

Benyong Chen, Zhejiang University of Science and Technology

(China)

Hongqiang Chen, GE Global Research (United States)

Daoxin Dai, Zhejiang University (China)

Qionghai Dai, Tsinghua University (China)

Qihuang Gong, Peking University (China)

Ying Gu, Chinese PLA General Hospital (China) 
Guang-Can Guo, University of Science and Technology of China (China)

Byoung Seung Ham, Gwangju Institute of Science and Technology (Korea, Republic of)

Sen Han, University of Shanghai for Science and Technology (China) and Suzhou H\&L Instruments, LLC (China)

Zuyuan He, Shanghai Jiao Tong University (China)

Werner H. Hofmann, Technische Universität Berlin (Germany)

Minghui Hong, National University of Singapore (Singapore)

Bahram Jalali, University of California, Los Angeles (United States)

Satoshi Kawata, Osaka University (Japan)

Baojun Li, Jinan University (China)

Ming Li, Institute of Semiconductors, CAS (China)

Ruxin Li, Shanghai Institute of Optics and Fine Mechanics (China)

Xingde Li, Johns Hopkins University (United States)

Jian Liu, PolarOnyx, Inc. (United States)

Tiegen Liu, Tianjin University (China)

Yongfeng Lu, University of Nebraska-Lincoln (United States)

Qingming Luo, Huazhong University of Science and Technology (China)

Gang-Ding Peng, The University of New South Wales (Australia)

Osamu Matoba, Kobe University (Japan)

Min Qiu, Westlake University (China)

Yuji Sano, ImPACT (Japan)

Yunlong Sheng, Université Laval (Canada)

Kebin Shi, Peking University (China)

Tsutomu Shimura, The University of Tokyo (Japan)

Upendra N. Singh, NASA Langley Research Center (United States)

Michael G. Somekh, Shenzhen University (China)

Yuguo Tang, Suzhou Institute of Biomedical Engineering and

Technology (China)

Masahiko Tani, University of Fukui (Japan)

Limin Tong, Zhejiang University (China)

Kazumi Wada, Massachusetts Institute of Technology (United States)

Yongtian Wang, Beijing Institute of Technology (China)

Rongshi Xiao, Beijing University of Technology (China)

Hongxing $\mathbf{X u}$, Wuhan University (China)

Jianhua Yao, Zhejiang University of Technology (China)

Toru Yoshizawa, Tokyo University of Agriculture and Technology (Japan) and 3D Associates, Inc. (Japan)

Changyuan Yu, The Hong Kong Polytechnic University

(Hong Kong, China)

Xiao-Cong Yuan, Shenzhen University (China)

Cunlin Zhang, Capital Normal University (China)

Song Zhang, Purdue University (United States)

Xi-Cheng Zhang, University of Rochester (United States) 
Xinliang Zhang, Wuhan National Laboratory for Optoelectronics

(China)

Xuping Zhang, Nanjing University (China)

Zhenrong Zheng, Zhejiang University (China)

Changhe Zhou, Shanghai Institute of Optics and Fine Mechanics (China)

Zhiping Zhou, Peking University (China)

Dan Zhu, Huazhong University of Science and Technology (China)

Ning Hua Zhu, Institute of Semiconductors, CAS (China) 


\title{
Conference Committee
}

\author{
Symposium Chairs
}

Jacobus M. Oschmann, Ball Aerospace (United States)

Qihuang Gong, Peking University (China)

Conference Chairs

Ming Li, Institute of Semiconductors (China)

Bahram Jalali, University of California, Los Angeles (United States)

Mohammad Hossein Asghari, Loyola Marymount University

(United States) and Tachyonics Inc. (United States)

Conference Program Committee

Hongwei Chen, Tsinghua University (China)

Xiangfei Chen, Nanjing University (China)

Hao Chi, Zhejiang University (China)

Yitang Dai, Tsinghua University (China)

Christophe Dorrer, University of Rochester (United States)

Shiming Gao, Zhejiang University (China)

Xiaoshun Jiang, Nanjing University (China)

Chanju Kim, Advanced Photonics Research Institute

(Korea, Republic of)

Yasushi Kondo, Shimadzu Corporation (Japan)

Hongpu Li, Shizuoka University (Japan)

Yong Liu, University of Electronic Science and Technology of China (China)

Asad M. Madni, University of California, Los Angeles (United States)

Kayvan R. Niazi, NantWorks, LLC (United States)

Tatsutoshi Shioda, Saitama University (Japan)

Daniel R. Solli, Ocunext Inc. (United States)

Yikai Su, Shanghai Jiao Tong University (China)

Kevin K. Tsia, The University of Hong Kong (Hong Kong, China)

Sergei K. Turitsyn, Aston University (United Kingdom)

Chao Wang, University of Kent (United Kingdom)

Jian Wang, Huazhong University of Science and Technology (China)

Ming Wang, Nanjing Normal University (China)

Xu Wang, Heriot-Watt University (United Kingdom)

Kun Xu, Beijing University of Posts and Telecommunications (China)

Lianshan Yan, Southwest Jiaotong University (China)

Akio Yazaki, Hitachi, Ltd. (Japan)

Changyuan Yu, The Hong Kong Polytechnic University (Singapore) 
Xinliang Zhang, Wuhan National Laboratory for Optoelectronics (China)

Xiaoping Zheng, Tsinghua University (China)

Xihua Zou, Southwest Jiaotong University (China)

\section{Session Chairs}

1 Optoelectronic Devices and Applications

Pengfei Wang, Harbin Engineering University (China)

2 Microwave Photonics for Measurement

Hongwei Chen, Tsinghua University (China)

3 Fiber Optic Sensing and Measurement

Wangzhe Li, Institute of Electronics, Chinese Academy of Sciences (China)

4 Ultrafast Optical Spectroscopy and Measurement

Hao Chi, Zhejiang University (China) 